\title{
Partial-Data Interpolation Method for Arc Handling in a Computed Tomography Scanner
}

Jaisingh Rajwade

Philips Healthcare, jai482@gmail.com

Lester Miller

Philips Healthcare, lester.miller@philips.com

Follow this and ad'ditional works at: https://engagedscholarship.csuohio.edu/enece_facpub

Cnietrtof Simentectrical and Computer Engineering Commons Cleveland State University, d.j.simon@csuohio,edu

How does access to this work benefit you? Let us know!

\section{Publisher's Statement}

NOTICE: this is the author's version of a work that was accepted for publication in Computerized Medical Imaging and Graphics. Changes resulting from the publishing process, such as peer review, editing, corrections, structural formatting, and other quality control mechanisms may not be reflected in this document. Changes may have been made to this work since it was submitted for publication. A definitive version was subsequently published in Computerized Medical Imaging and Graphics, 35, 5, (07-01-2012); 10.1016/j.compmedimag.2012.04.004

\section{Original Citation}

Jaisingh Rajwade, Lester Miller, Dan Simon. (2012) Partial-data interpolation method for arc handling in a computed tomography scanner. Computerized Medical Imaging and Graphics, 36(5), 387-395. doi:

10.1016/j.compmedimag.2012.04.004

\section{Repository Citation}

Rajwade, Jaisingh; Miller, Lester; and Simon, Daniel J., "Partial-Data Interpolation Method for Arc Handling in a Computed Tomography Scanner" (2012). Electrical Engineering \& Computer Science Faculty Publications. 137. https://engagedscholarship.csuohio.edu/enece_facpub/137

This Article is brought to you for free and open access by the Electrical Engineering \& Computer Science Department at EngagedScholarship@CSU. It has been accepted for inclusion in Electrical Engineering \& Computer Science Faculty Publications by an authorized administrator of EngagedScholarship@CSU. For more information, please contact library.es@csuohio.edu. 


\title{
Partial-data interpolation method for arc handling in a computed tomography scanner
}

\author{
Jaisingh Rajwade ${ }^{\mathrm{a}, *}$, Lester Miller ${ }^{\mathrm{b}, 1}$, Dan Simon ${ }^{\mathrm{c}, 2}$ \\ a Philips Healthcare, 595 Miner Road, Highland Heights, $\mathrm{OH} 44143$, United States \\ b Philips Healthcare, 555 North Commerce Street, Aurora, IL. 60504, United States \\ 'Cleveland State University, 2121 Euclid Avenue, Cleveland, $\mathrm{OH} 44115$, United States
}

\section{Introduction}

Despite numerous methods of image reconstruction developed over the years in computed tomography (CT), there has been limited work in interpolation methods specific to X-ray tube arcing. This is primarily due to the fact that standard interpolation methods have typically been effective in eliminating artifacts caused by data loss due to arcing. However, with the continuous drive from the imaging field to have faster scanners with short image acquisition times, adverse effects due to arcing are becoming more significant. Short arc durations are now significant contributors to larger portions of the image data than before and the need to address this is more relevant.

We reviewed existing interpolation methods to look at different ways of dealing with missing data in reconstruction algorithms used in CT since this is also the underlying purpose of our proposed method. There have been methods developed to reduce artifacts caused by missing data such as those proposed for metal artifact reduction $[1,2]$. These methods successfully lowered artifacts in the

\footnotetext{
* Corresponding author. Tel.: +1 4404834603 ; fax: +216 6594111. E-mail addresses: jai482@gmail.com (J. Rajwade), lester.miller@philips.com (L. Miller), d.j.simon@csuohio.edu (D.Simon).

1 Tel.: +16305852181 .

2 Tel.: +12166875407 .
}

images but at times introduced issues like blurring and additional artifacts. Our method specifically addresses reducing artifacts caused in CT scanners by arcing of X-ray tubes. Our premise is that errors will be less if corrected data is used for imaging during an arc rather than assuming a continuous trend and filling in missing data (interpolating).

Section 1.1 of this introduction gives a brief review of arc detection in CT systems. Section 1.2 reviews current methods of dealing with missing data due to arcs. Section 1.3 provides a conceptual overview of our proposed method for reducing artifacts due to arcing. Section 1.4 discusses some of the benefits of our proposed approach to artifact reduction.

\subsection{Arc detection in the $X$-ray $C T$ system}

Fig. 1 shows a method used in a Philips CT scanner to detect arcing in tubes. The power supply detects an arc and passes the information to the detection system, which then handles it using interpolation to minimize detrimental effects on image quality.

The tube arc signal becomes active every time the voltage drops below the $90 \%$ set point. It remains active until the voltage is above the $90 \%$ value again. This signal tells the image reconstruction system when and for how long to perform interpolation during the scan. If an arc occurs during a patient scan, the shutdown of tube power results in a momentary loss of X-rays, and therefore a loss of 


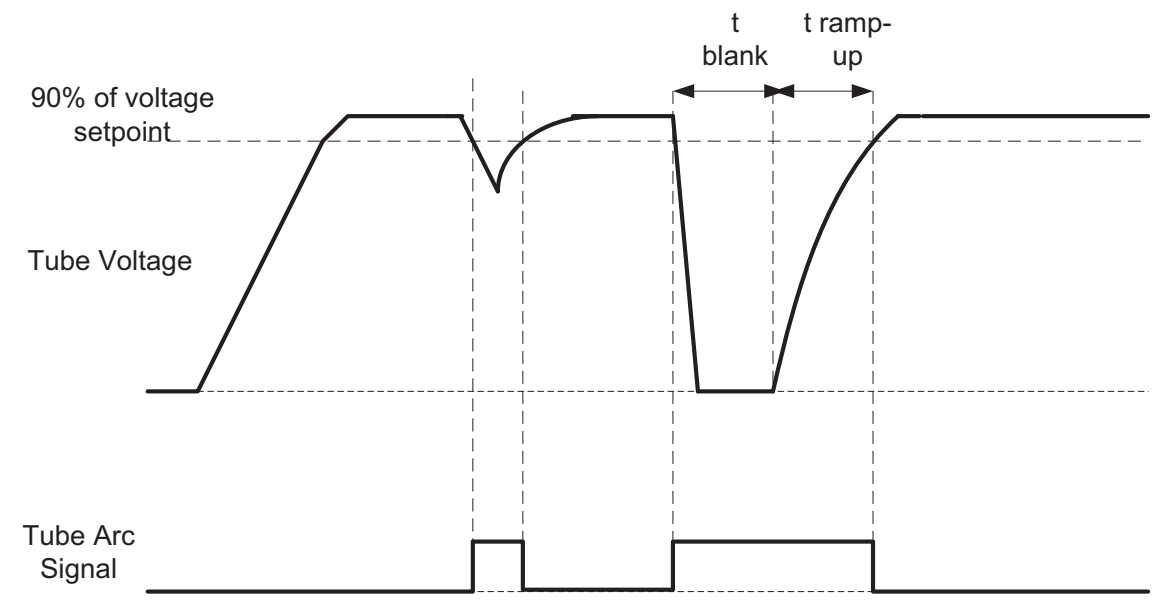

Fig. 1. Arc detection and reporting in X-ray power supply.

imaging data. The length of this momentary period is determined by the time it takes for the power supply's voltage source to recover, which may be on the order of several milliseconds.

Typical image computation or reconstruction algorithms will detect these arc events, and interpolate data during the shutdown period to continue with the patient scan $[3,4]$. This method can be acceptable from an imaging point of view depending on how long the shutdown period of the system may be and the number of arcs that occur during it. However, the tube voltage recovery time becomes relatively larger compared to scan time in scanners that have high rotation and reconstruction speeds and frequently the artifacts get worse to a level that the image is not acceptable and the patient must be scanned again [5].

\subsection{Limitations of current interpolation methods}

When a CT scan is performed, data obtained during the scan is used to reconstruct images for the user. The data contains certain information about the scan in the file header, such as the time and position of the X-ray beam when the data is collected. This header also contains a status signal that has information about arcs, as shown in Fig. 1. Common reconstruction algorithms discard data that corresponds to the arc duration and interpolate over it. These methods are usually simple linear or Lagrange methods of interpolation $[5,6]$ depending on the manufacturer.

Our experiments to evaluate limitations of current methods consisted of taking scan data, activating arc status in the header information for some amounts of time, and then reconstructing the image with this data, thus giving an image that would be obtained with real arcing in the scanner. A large number of these simulations were performed to understand the effect of arcing on images with respect to magnitude, duration and other factors.

The simulations were done using the reconstruction routine used by the development team at Philips Healthcare and was implemented in Matlab ${ }^{\circledR}$. Note that the goal of this project was not to develop a new interpolation algorithm, but to provide a new translation method to generate data during where there would be no data available. This is why we used existing reconstruction methods to do simulations and verifications.

The reconstruction results obtained using current interpolation methods were analyzed to identify areas that could use further improvements. The scans shown in Fig. 2 were done on a 64 slice Philips Brilliance ${ }^{\mathrm{TM}} \mathrm{CT}$ scanner. The left image is without any arcs, the center image shows the same phantom with 10 arcs, and the right image is the mathematical difference of the two, which shows the artifact caused by the arcing more clearly. The difference image shows undesired streaks and marks (artifacts), caused by arcs. Note that arcing changes the photon output of the X-ray tube, which affects the projections that are acquired by the detectors. The projections, when processed by the image reconstruction system, result in imperfections in image quality. The ultimate acceptability of the images is defined by medical practitioners that use the scanners, but we are able to see from the difference images that interpolation of data during arcing does not eliminate artifacts completely.

\subsection{Proposed implementation}

Fig. 3 shows a standard arc handling method where data is interpolated over the entire arc duration. We chose to use a common interpolation method that was readily available. The two-data point interpolation shown here is commonly used in industry and is found to be effective in most cases. Fig. 4 shows the proposed method of partial-data interpolation where we use the new algorithm to translate or correct measured data to its equivalent at the programmed voltage during the tail part of the arc event and perform two-point interpolation over the remaining duration. It shows the shutdown period of the power supply where the voltage is zero after which it recovers to its set point. Image data collected during the latter part of the recovery is corrected and used.

Our premise again is that corrected data is more representative of human anatomy than when only interpolation is used without correction, especially when the attenuation is changing rapidly during the arc.

The method to find which points to correct during the arc period was based on selecting a certain threshold of voltage value, which in our case was approximately $60 \%$ of programmed value and then correcting data only above that voltage level. We used $60 \%$ because we found through experimentation that the signal below $60 \%$ would make the signal to noise ratio very low. The $60-90 \%$ range allows for the correction of up to $30 \%$ of the data in the total arc period. The proposed partial-data recovery is performed in-line with standard interpolation and is a complementary method rather than a replacement for the standard interpolation.

\subsection{Potential benefits of partial-data interpolation method}

This research introduces a new method of correcting for data loss during arcing on a CT scanner which could provide the following benefits. 

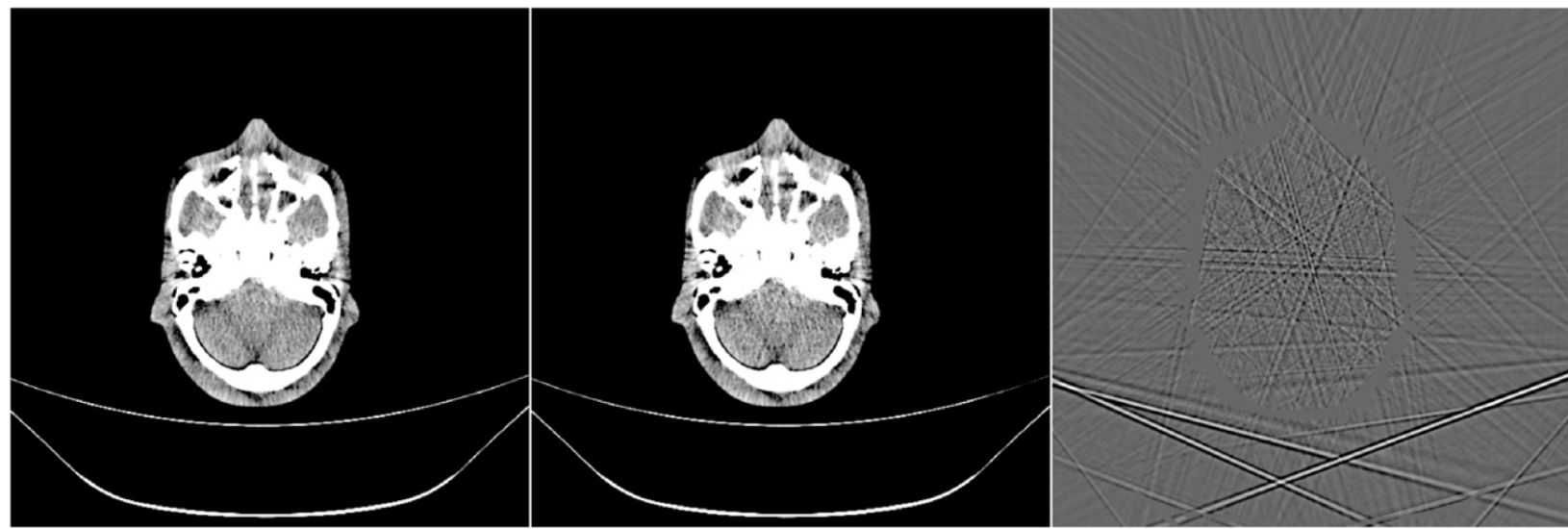

Fig. 2. Head phantom images: left image, no arcs; center image, 10 arcs using interpolation correction; right image, difference between left and center images.

- Use of the partial-data interpolation method results in improvement in image quality during tube arcing.

- Sometimes scanning has to be repeated because of arc induced artifacts. This method could reduce the possibility of repeating the scan by reducing artifacts caused during part of the scan. This could mean a $50 \%$ lower radiation exposure to a patient in such cases by not having to repeat the scan when arcing occurs.

- X-ray tube arcing rates typically increase when the tubes near end of their life. This method could help extend some of the tube life by allowing use of the scanner while a replacement is received.

- Improved arc handling and minimization of its effects can prove to be a competitive edge for a scanner manufacturer.

Section 2 details the implementation sequence of the new algorithm, Section 3 reviews the results obtained with the algorithm implementation and Section 4 draws conclusions from the study.

\section{Methods}

\subsection{Real-time measurement of voltage using the detector system}

Though the voltage feedback of the power supply is available from the system electronics, it is not fast enough in some scanners (due to hardware propagation delays) to provide real-time information. We therefore need to perform voltage measurement simultaneous with image data acquisition. We use a material with known attenuation characteristics (copper) to make relative measurements on the detectors and relate it to tube voltage. Even though this is a known method of voltage detection [7], it is not typically implemented in CT scanners since there has not, prior to this research, been a need for real-time voltage feedback from the detectors.

Using an X-ray attenuation simulation program, we calculated the effects of different thicknesses of copper on the signal level
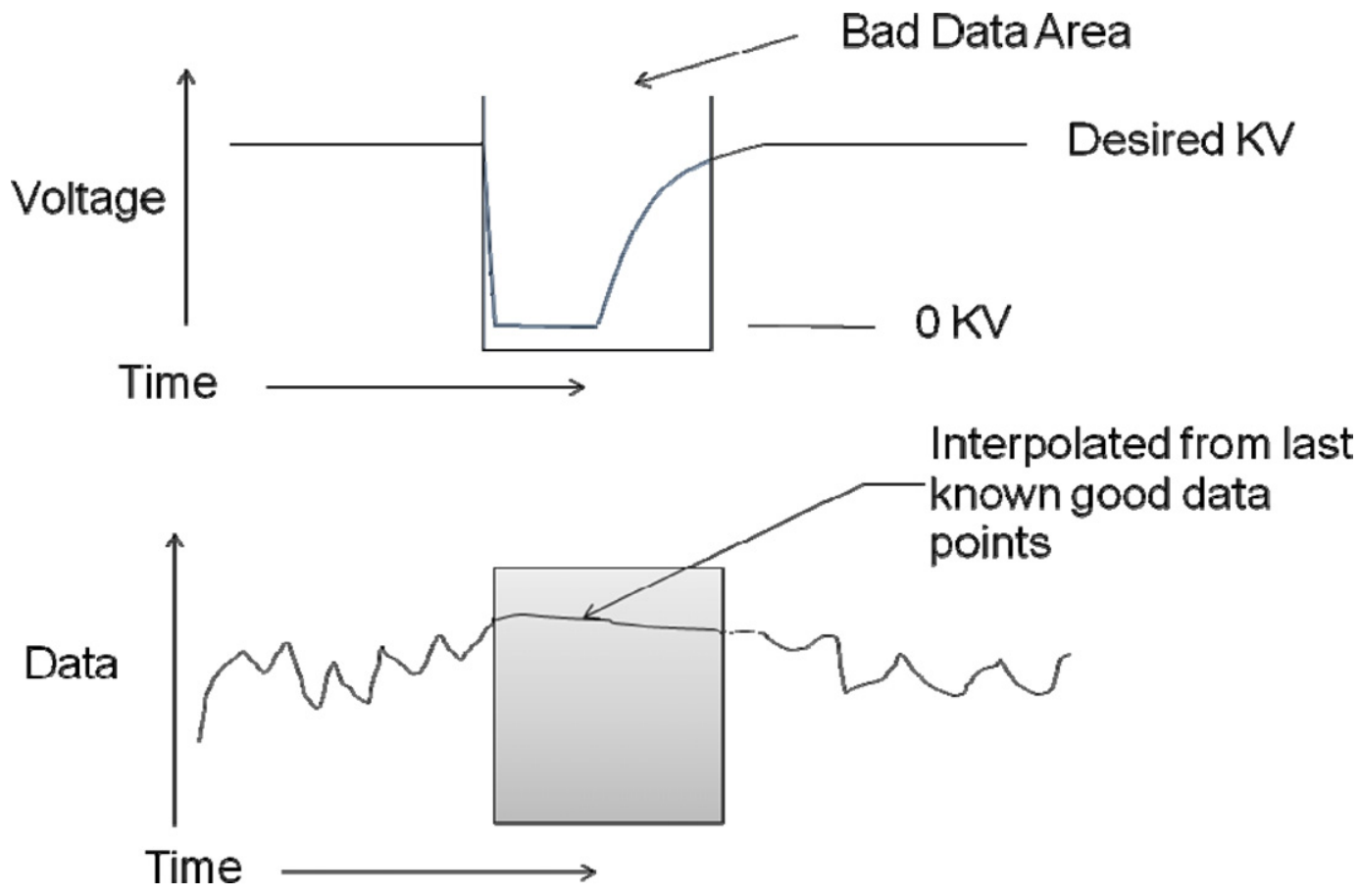

Fig. 3. Standard arc handling. 

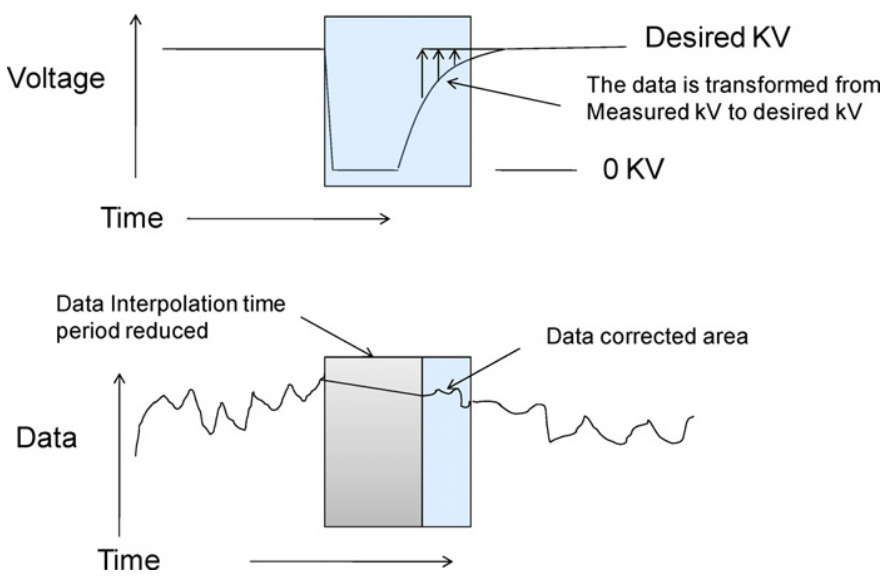

Fig. 4. Proposed implementation for partial-data interpolation.

seen by the detectors in a CT scanner. Copper was chosen because it is a good X-ray attenuating material, it is easy to make into thin sheets to place on the CT detectors, and is relatively inexpensive and readily available. Other materials like tin or molybdenum could also be used for this purpose [7]. The simulation program generates a spectrum of the X-rays coming out of a tube and then uses the different materials in the beam path to attenuate these rays on their way to the detectors.

We also made measurements on a scanner with different thicknesses of copper placed over the detectors and were able to prove that the simulation program was a good representation of the real scanner. The measurement was done by placing rectangular pieces of $1 \mathrm{~mm}$ copper sheets over the detectors on the scanner and making scans. The data was then analyzed and the area which showed the attenuation due to the copper sheet was noted by specific detector locations. We then took the attenuation values at points under this copper covered area and calculated the ratio with values that were not covered by the sheets.

Attenuation ratio is represented as follows:

$\frac{I}{I_{0}}=e^{-\mu t}$

where $I_{0}$ is the original intensity of the X-ray beam; $I$ is the intensity of the X-ray beam; $e$ is Euler's number; $\mu$ is the effective attenuation coefficient for polychromatic X-ray beam; $t$ is the thickness of attenuating material.

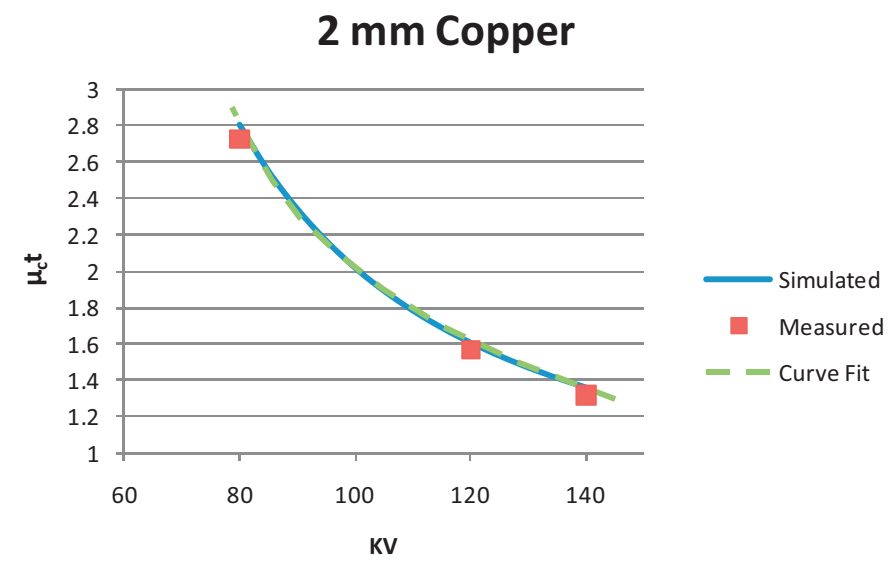

Fig. 6. Relationship between voltage and $\mu_{c} t$ (simulated), measured and curve fit (Eq. (3)) for $2 \mathrm{~mm}$ copper thickness.

Based on Eq. (1), the expression for the effective $\mu t$ (logged attenuation ratio) is derived as follows:

$\mu t=-\ln \frac{I}{I_{0}}$

All calculations and measurements for this project were done using the logged attenuation ratio $\mu t$, shown in Eq. (2). Since we use $\mu$ to denote attenuation coefficients for both copper and water in this paper, we will henceforth refer to them as $\mu_{c}$ for copper and $\mu_{w}$ for water. Fig. 5 shows the relationship between simulated $\mu_{c} t$ and voltage for different thicknesses of copper.

We chose to use $2 \mathrm{~mm}$ of copper to stay in a low attenuation range to keep the signal level above $1 \%$ for a good signal to noise ratio and at the same time have a good variation for different voltages. This $1 \%$ threshold is an empirically chosen level below which the signal is too low to be useful for our measurements. Also, it makes the implementation simpler to use an attenuation curve with a reasonable slope so as to get clear and distinctive voltage estimation. Fig. 6 shows the simulated, measured and fitted curves for $2 \mathrm{~mm}$ of copper, which highlights the closeness of the simulated data to the measured data.

For the implementation of the partial-data algorithm, we required a function that fitted the measured and simulated data to determine the tube voltage. Based on the $\mu_{c} t$ to voltage relationship shown in Fig. 5, Eq. (3) was derived, using a polynomial fit function, for the curve for $2 \mathrm{~mm}$ of copper. We found that a fourth

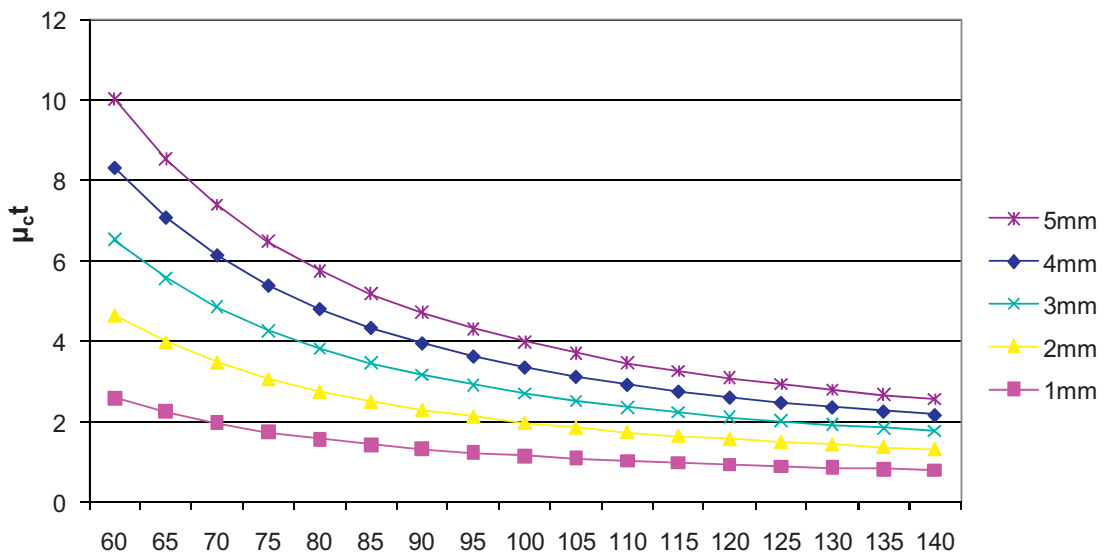

KV

Fig. 5. Relationship between voltage and $\mu_{c} t$ (simulated) for different copper thicknesses. 


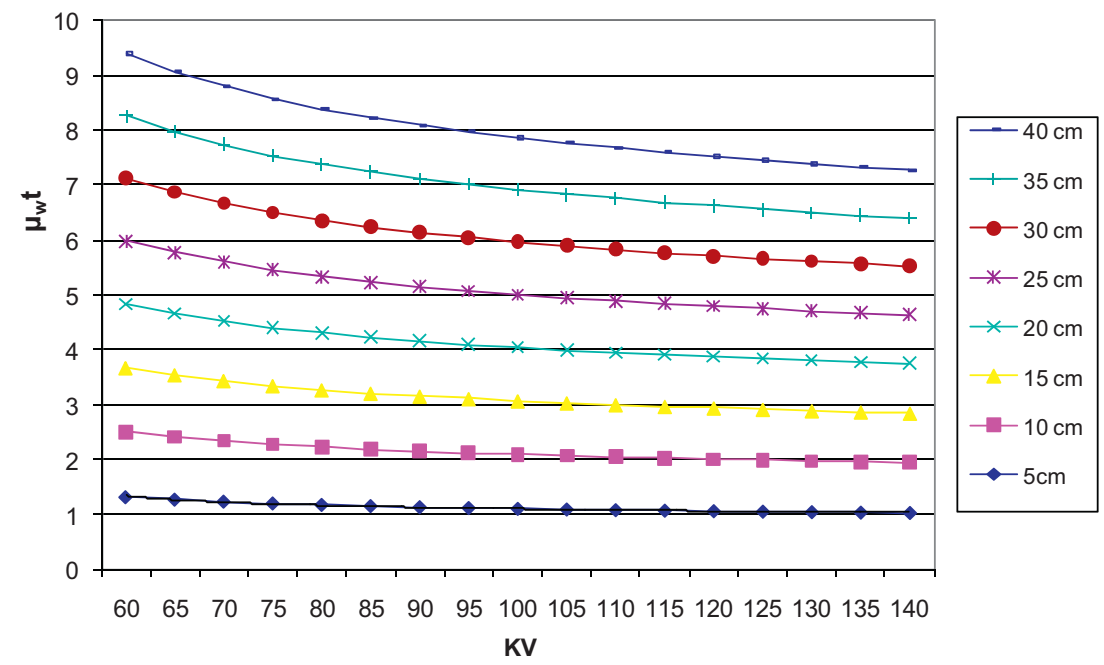

Fig. 7. Relationship between voltage and $\mu_{w} t$ for different thicknesses of water (simulated).

order polynomial gave good representation of the curve with low error. This equation represents the real data with an accuracy of approximately $3 \%$ and is also shown as the curve fit in Fig. 6.

$$
\begin{aligned}
v & =1.7443\left(\mu_{c} t\right)^{4}-24.755\left(\mu_{c} t\right)^{3}+132.3188\left(\mu_{c} t\right)^{2} \\
& -327.8174\left(\mu_{c} t\right)+396.9295
\end{aligned}
$$

where $v$ is the tube voltage. This equation is used to calculate the voltages (using $2 \mathrm{~mm}$ of copper) along the rising curve after the arc.

\subsection{Partial-data interpolation algorithm}

This section describes the primary contribution of this paper. Data is collected during the rising voltage after an arc at values below the nominal (or programmed) value, while simultaneously estimating the voltage value using the method developed in Section 2.1. This voltage value is then used to correct the image data, from which the reconstruction can then be performed. Calculating the image data at the desired voltage involves translating the attenuation created by a particular object (or part of the human body) at the measured voltage to an attenuation that is equivalent to that caused by the body at the desired voltage. Our assumption for this research was that for a first order correction, the human body can be considered to be made of water. Fig. 7 shows the simulation of attenuation ratios for water of different thickness levels. It provides for a $\mu_{w} t$ measured at a particular voltage (low, recovering) to be translated to the $\mu_{w} t$ value we would expect at the desired (programmed) voltage.

Note that the measurements shown in Fig. 7 were made without the standard beam-hardening compensator in the X-ray path to simplify our process. This paper describes a preliminary implementation to prove the proposed concept and beam hardening will need to be considered for full system implementation. Our method can also be applied after the beam hardening correction is done, so beam hardening should not affect its improvement of the interpolation method. For the partial-data algorithm implementation, the data shown in Fig. 7 was fitted to a convenient expression, shown in Eq. (4).

$\mu_{w} t=\frac{q}{(v-b)^{0.5}}+C$

where $v$ is the voltage and $a, b$ and $c$ are constants to be determined. We used trial and error to arrive at the structure for Eq. (4). For each thickness shown in Fig. 7 (5 cm through $40 \mathrm{~cm}), a, b$ and $c$ were determined using the Newton-Raphson method in Mathcad ${ }^{\circledR}$. Then each $a, b$ and $c$ were fitted to first and second order polynomials in terms of $t$. We then replaced $a, b$ and $c$ in terms of thickness $t$, in Eq. (4) to get $\mu_{w}$ as shown in Eq. (5).

$$
\begin{aligned}
& \mu_{w} t=\left(\frac{\left(0.0026 t^{2}+0.5191 t+0.3801\right)}{\left(V+0.0015 t^{2}+0.0620 t-33.5132\right)^{0.5}}\right) \\
& +0.1181 t+0.2064
\end{aligned}
$$

where $t$ is the thickness of the relevant part of the scanned object. This equation was found to provide results with an average accuracy of approximately $2.5 \%$ when compared with the data.

The partial data algorithm can then be implemented in the following steps and is also illustrated in Fig. 8.

A. Scan an object during an arc event and collect data which contains the $\mu_{c} t_{c}$ (copper) and $\mu_{w} t_{w}$ (water) data needed for subsequent calculations.

B. Solve for voltage measured during the arc using Eq. (3).

C. $\mu_{w} t_{w}$ is the phantom data, which is based on our approximation that the body equals water.

D. Using the voltage obtained from step B, use Eq. (5) to solve the thickness of water ( $t_{w 2}$ in Fig. 8 ) at the voltage $\mathrm{kV}_{\mathrm{m}}$ and $\mu_{w} t_{w}$.

E. Use thickness of water and desired voltage to solve for corrected $\mu_{w} t_{w}$.

F. Repeat steps B through E for all points measured on the rising voltage curve.

A more complete description of the method is provided in Appendix A.

\subsection{Experimental setup and validation of the algorithm}

Fig. 9 shows the setup used in the implementation and validation of the partial-data algorithm. It shows the copper strips placed over a small number of detectors at one end of the array, which serve to measure real-time voltage. The correction is implemented after collecting data from the scan. The phantom is the object being imaged and the images obtained are shown in Section 3.

Note that the copper strips in this case have been placed on the scanner detectors for convenience; they could alternatively be 


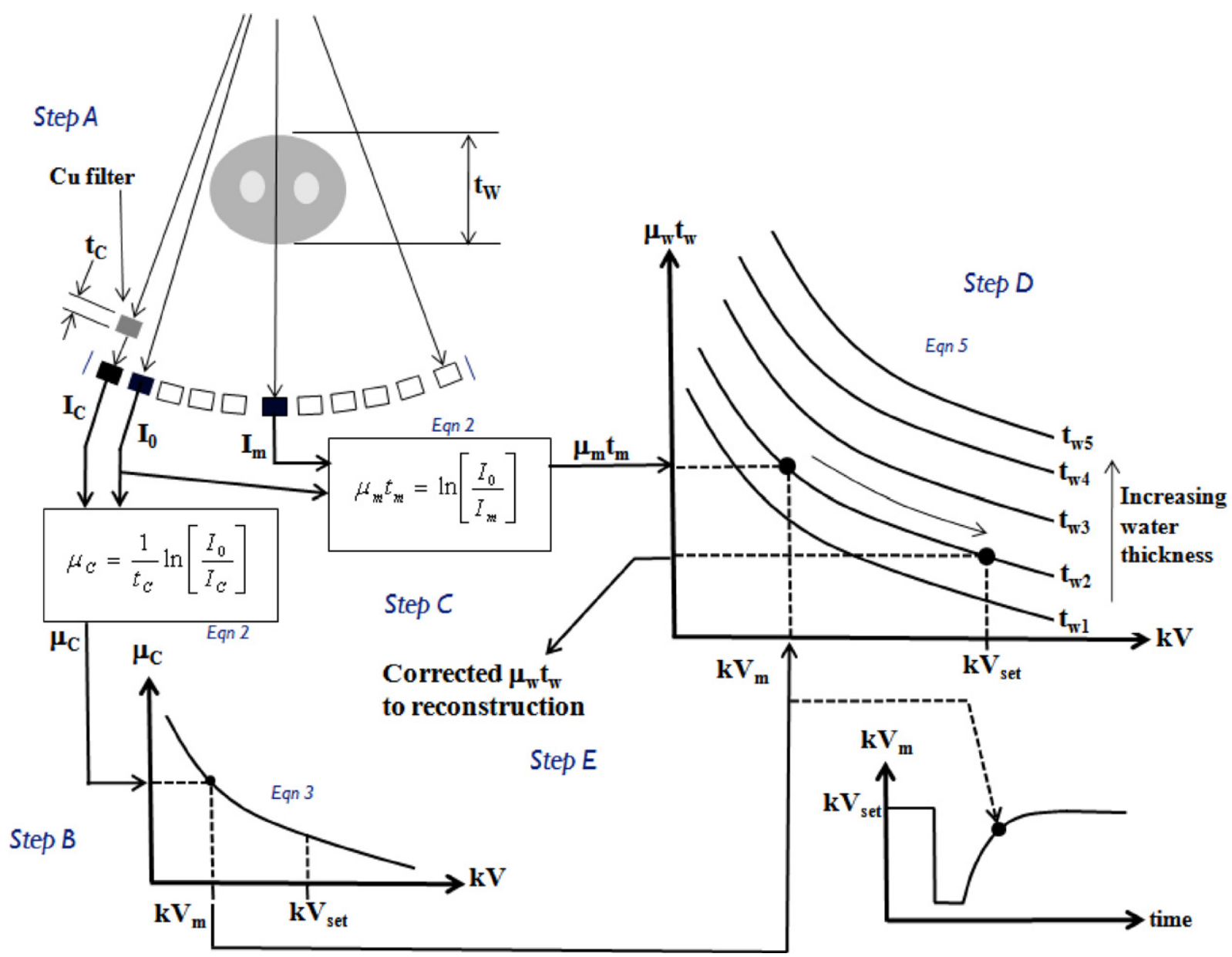

Fig. 8. Illustration of the partial-data algorithm implementation.

placed on detectors in the collimator assembly to keep them out of the path of the detectors in system implementation.

Since X-ray tube arcing is an unpredictable and uncontrollable phenomenon, the verification has been done using simulated code on a scanner which emulates arcing. Scans were performed using this arcing software followed by reconstruction on data collected from these scans.

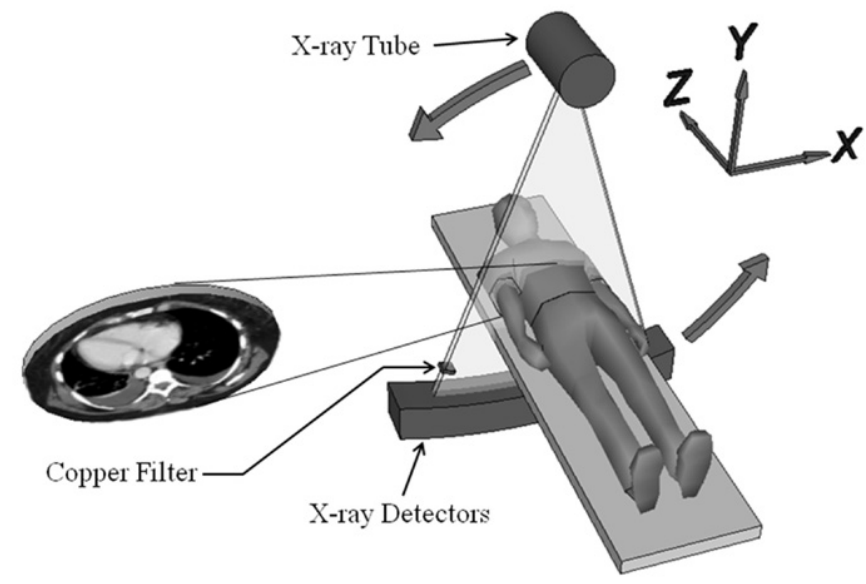

Fig. 9. Location of copper filter at edge of CT detector array and unblocked by scanned object.

\subsection{Implementation method}

The partial data algorithm was implemented on data that was collected on a Philips Brilliance $\mathrm{iCT}^{\mathrm{TM}}, 256$ slice scanner using arc simulation software, which causes the power supply to respond as it would during a real arc. A total of $2 \mathrm{~mm}$ thickness of copper (two strips of $1 \mathrm{~mm}$ each) was placed in the X-ray path to detect the rising voltage level during an arc, and the data was then processed using both standard interpolation and partial data algorithm. All were axial scans performed at $120 \mathrm{kV}, 919 \mathrm{~mA}$, and $0.27 \mathrm{~s}$ duration at $220 \mathrm{rpm}$. Tube arcing during normal operation is random. However, typical arcing rates based on our experience are below $0.1 \%$ of total scan-seconds. The special code was made to cause an arc status every $90 \mathrm{~ms}$, so three arcs were simulated in the $270 \mathrm{~ms}$ scan period. The power supply, on seeing this arc status, would respond like it does to a real arc, by shutting down the voltage production for $500 \mu \mathrm{s}$ and then bringing up the voltage to the requested value (another $500 \mu \mathrm{s}$ ), thus creating an arc period of $1 \mathrm{~ms}$. Each arc's duration was $1 \mathrm{~ms}$, so this corresponded to $3 \mathrm{~ms}$ over a total period of one rotation ( $270 \mathrm{~ms}$ in our case). This is an arc percentage of $1.1 \%$.

The partial data algorithm was implemented in Matlab code, which processed the data from the scanner, using the arc status in the data file header to select views to be corrected during arcing. The number of bad data views that an arc could cause depends on the magnitude and duration of the arc, but the system that we used can typically handle up to 15 bad views before the image becomes too bad to use for diagnostic purposes. In our case the arc duration 


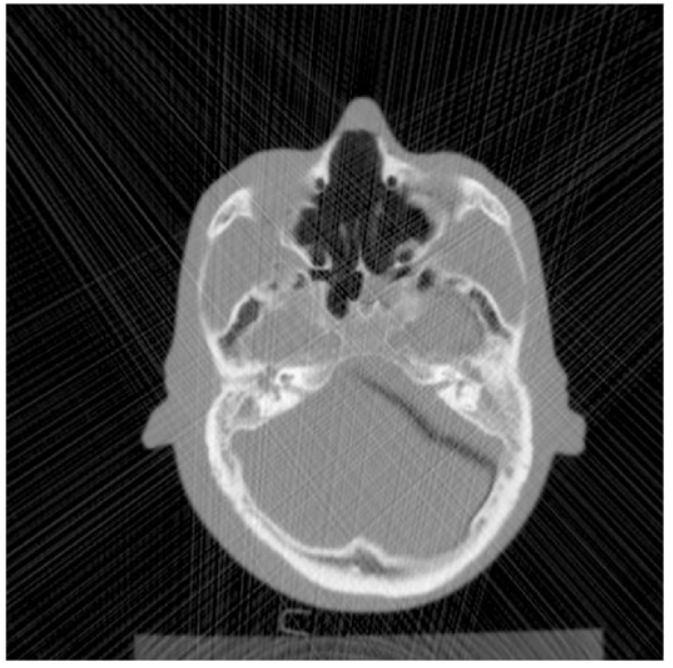

Fig. 10. No interpolation showing streak artifacts.

caused 10 bad views. The object used was a head phantom. Radiology phantoms are commonly used in the medical arena to test imaging systems due to their likeness to the human body [8].

\section{Results}

The resulting images are shown in Figs. 10 through 12 . The display window used in all figures was 0-100 Hounsfield Units (HU), viewing level was set at $1100 \mathrm{HU}$, so we are viewing the images from 1050 to $1150 \mathrm{HU}$. Fig. 13 shows the difference image of the two methods, which highlights that the partial-data method corrects streaks that are not properly corrected by the standard interpolation-only method.

Note that a uniform region containing artifacts was chosen as the region of interest. The standard deviation in these regions shows which image has the lower variation or noise level. It can be seen that the partial data algorithm shows an improvement over the standard interpolation method. This difference is only small in this case ( 8.99 vs. $8.64 \mathrm{HU})$ but it is an improvement over the interpolation method and will be even more pronounced in scanners with higher rotation speeds. The standard deviation in an image without any arcs for the same region was $5.10 \mathrm{HU}$.

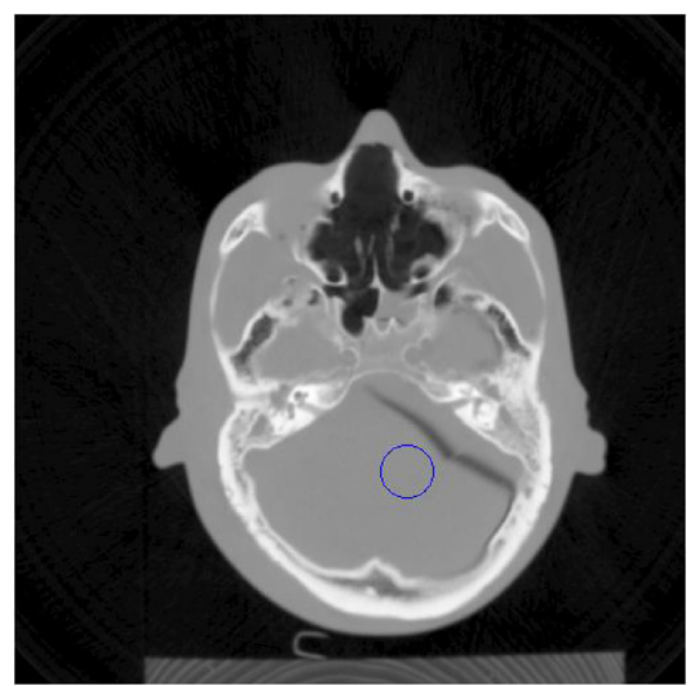

Fig. 11. Head phantom using standard interpolation with highlighted region of interest (ROI). Standard deviation $=8.99 \mathrm{HU}$.

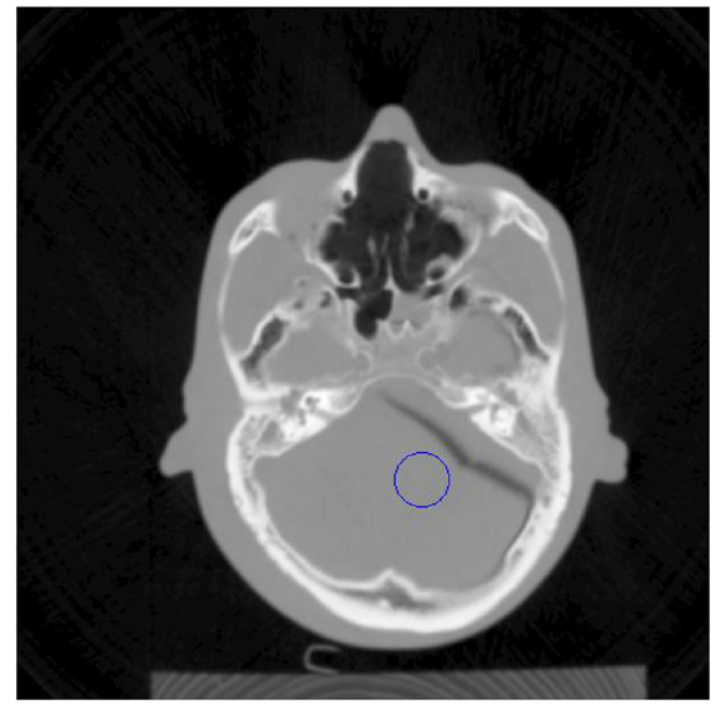

Fig. 12. Head phantom using partial data interpolation with highlighted ROI. Standard deviation $=8.64 \mathrm{HU}$.

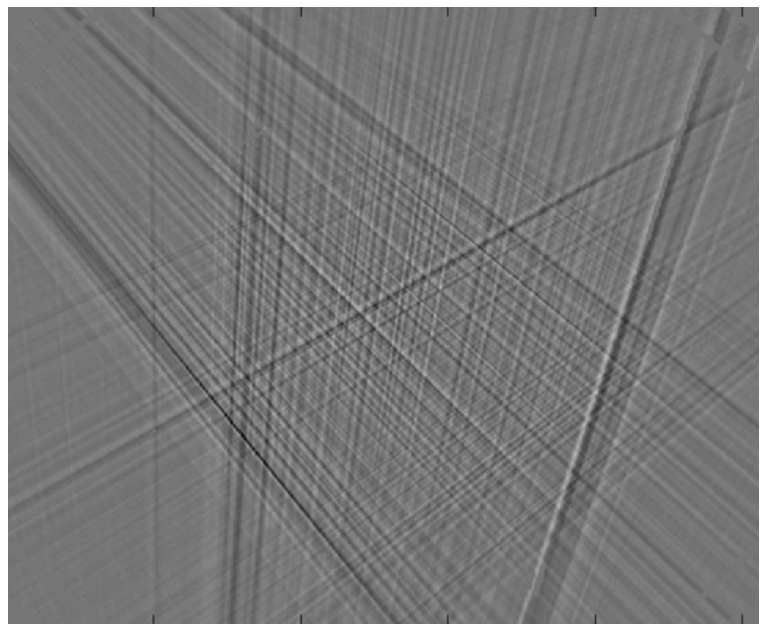

Fig. 13. Difference image of Figs. 11 and 12.

Multiple sets of images were reconstructed using both methods. Different locations for ROI's were selected. Results are shown in Table 1.

The comparison shows that there is a lower standard deviation in the partial data interpolated images, which indicates fewer artifacts. Even though there is no single measure for defining image quality in CT scanning, there are several different quantitative evaluations that have been developed. Standard deviation is considered

Table 1

Statistics for head phantom from multiple scans using standard and partial-data methods.

\begin{tabular}{llll}
\hline $\begin{array}{l}\text { Scan } \\
\text { num- } \\
\text { ber }\end{array}$ & $\begin{array}{l}\text { Std deviation } \\
\text { using standard } \\
\text { interpolation (HU) }\end{array}$ & $\begin{array}{l}\text { Std deviation using } \\
\text { partial-data } \\
\text { interpolation }(\mathrm{HU})\end{array}$ & $\begin{array}{l}\text { Improvement of } \\
\text { partial-data over } \\
\text { standard } \\
\text { interpolation (\%) }\end{array}$ \\
\hline 1 & 8.99 & 8.64 & 3.88 \\
2 & 9.78 & 9.61 & 1.78 \\
3 & 8.13 & 7.31 & 10.02 \\
4 & 11.33 & 10.57 & 6.72 \\
5 & 8.00 & 6.98 & 12.75 \\
6 & 12.10 & 11.71 & 3.22 \\
\hline
\end{tabular}


Table 2

Scanner rotation speeds and their effects on data lost during arcing. We used 2400 views per rotation.

\begin{tabular}{llll}
\hline $\begin{array}{l}\text { Scanner } \\
\text { speed } \\
(\mathrm{rpm})\end{array}$ & $\begin{array}{l}\text { Time for one } \\
\text { rotation }(\mathrm{s})\end{array}$ & $\begin{array}{l}\text { Integration } \\
\text { period = rotation } \\
\text { time/\# of views in one } \\
\text { rotation }(\mu \mathrm{s})\end{array}$ & $\begin{array}{l}\text { Angular data lost } \\
\text { during } 1 \mathrm{~ms} \\
\text { arc }=1 \mathrm{~ms} / \text { rotation } \\
\text { time } \times 360\left(^{\circ}\right)\end{array}$ \\
\hline 120 & 0.50 & 208 & 0.72 \\
180 & 0.33 & 137 & 1.09 \\
200 & 0.30 & 125 & 1.2 \\
220 & 0.27 & 112 & 1.33 \\
300 & 0.20 & 83 & 1.8 \\
\hline
\end{tabular}

a general figure of merit for artifacts as shown in [9], which relates the variance $\left(\sigma^{2}\right)$ to the image quality.

To quantify the effect that scan speed will have on how much data is lost or can be saved by this new method, we have compared some scenarios in Table 2. Assuming an arc lasts about $1 \mathrm{~ms}$ (time taken to recover from an arc in a Philips premium system today), and since one rotation is $360^{\circ}$, we calculate the amount of data lost during one rotation in terms of the angular data.

Since CT images are generated using data obtained during angular acquisition during a scan, any loss of angular data is a contributor to artifacts in images [5]. In the case of, for example, the $220 \mathrm{rpm}$ scan, we lose $1.33^{\circ}$ during each arc and each arc lasts at least $1 \mathrm{~ms}$, so if we have multiple arcs we can lose several degrees of data. However, using the partial-data interpolation method we can save up to $30 \%$ of the data (that is, data between $60 \%$ and $90 \%$ of the rising voltage) from the arc duration from being lost. If we have three contiguous arcs on the $220 \mathrm{rpm}$ scan, we can save $30 \%$ of $3 \times 1.33^{\circ}$, which is approximately $1.2^{\circ}$. One can see from Table 2 that if we save the same percentage in an even faster scan, like the $300 \mathrm{rpm}$ scan, we can save up to $1.6^{\circ}$ of data from being lost. This shows the significance that rotation speed has on the amount of data being recovered. Note that $120-220 \mathrm{rpm}$ scanners already exist in today's market. It is also known from market reports that at least one CT manufacturer is currently working on a $300 \mathrm{rpm}$ scanner [10] and others will follow suit soon afterwards.

\section{Conclusion}

A new method of interpolation has been proposed for handling arcing in an X-ray tube in a CT scanner. We collect image data while the tube voltage recovers to its programmed value after an arc, providing voltage corrected data during arcs (in combination with interpolation) as opposed to a method that uses only interpolation. Preliminary results show that this is an effective method and will enhance the performance of a high rotation speed scanner in arcing situations.

The algorithm was verified on a Philips Brilliance CT scanner during an arc event. The test was performed on a system with special software that simulated arc conditions by programming arcs into the system, which caused the voltage to switch off and turn on again after a short delay. We scanned a head phantom on a scanner with this arc code installed. We then reconstructed both data sets, one with a standard interpolation method and the other with our partial-data algorithm. We compared the two images by selecting a region of interest in the same location in both images, and running a statistical analysis on both. The region of interest chosen was one with maximum uniformity, so the standard deviation shows the variation in a region where the lower the deviation, the lesser the artifacts. In this comparison, the partial-data algorithm showed an improvement over the standard interpolation method.

This study is only the first step to provide a preliminary implementation of the proposed method. More work will need to be done to account for attenuation coefficient characterization of body parts like bone, tissue, fat and contrast agents. The experiment was done using water coefficients to prove that the concept works. Note that the results show tests performed on head phantoms, which are designed to be representative of non uniform characteristics of a human head. Other issues to consider would be to use more complex interpolation methods like Lagrange [5] or weighted multiple point interpolation in conjunction with partial-data interpolation to improve image results further. This study was performed with a futuristic view, and will become more relevant as the imaging industry pushes toward faster rotational and sampling speeds, for which arcing will manifest more adverse effects on images.

\section{Appendix A.}

Following are the detailed implementation steps of the partialdata interpolation method, as shown in Fig. 8.

A. Scan an object during an arc event and collect measured image data $\left(I_{m}\right)$, data from a detector covered with copper $\left(I_{c}\right)$ and data from an unblocked reference detector $\left(I_{0}\right)$. The data contains the $\mu_{c} t_{c}$ (copper) and $\mu_{w} t_{w}$ (water) needed for subsequent calculations.

B. Solve for voltage measured at a particular point $\left(\mathrm{kV}_{\mathrm{m}}\right)$ on the rising voltage curve using Eq. (3). We have $\mu_{c} t_{c}$ (measured) at the copper strip covered detectors.

C. Equate the $\mu_{m} t_{m}$ to $\mu_{w} t_{w}$ based on our approximation that the body equals water. $\mu_{m} t_{m}$ (measured) $=f(v$ (estimated), $t)$ as shown in Eq. (5).

D. Using the $\mu_{w} t_{w}$ from the image detector data and $\mathrm{kV}_{\mathrm{m}}$ obtained from step B, use Eq. (5) to solve for $t$ (estimated) using an iterative method. This is the thickness of water $\left(t_{w 2}\right.$ in Fig. 8) at the voltage $\mathrm{kV}_{\mathrm{m}}$ and $\mu_{w} t_{w}$.

E. Use this thickness of water to solve the following (also using Eq (5)):

$\mu_{w} t_{w} \quad$ (corrected $)=f(v($ programmed $), \quad t$ (estimated $\left.)\right) . \quad \mathrm{kV}_{\text {set }}$ shows the programmed voltage in Fig. 8 .

This is the translated $\mu_{w} t_{w}$ provided to the reconstruction system to fill in the missing data during the arc.

F. Repeat steps B through E for all points measured on the rising voltage curve.

\section{References}

[1] Watzke O, Kalendar WA. A pragmatic approach to metal artifact reduction in CT: merging of metal artifact reduced images. Eur J Radiol 2004;14(5):849-56.

[2] Betram M, Wiegert J, Schaefer D. Directional view interpolation for compensation of sparse angular sampling in cone-beam CT. IEEE Trans Med Imaging 2009;28(July (7)):1011-22.

[3] Honda T, Taguchi W. X-ray computed tomography apparatus. US Patent 64,49,337; 10 September 2002.

[4] Hsieh J, Schmidt J, Gordon C, Block J. Methods and apparatus for tube-spit correction. International Patent WO 02/43451 A2; 30 May 2002.

[5] Hsieh J. Computed tomography - principles, design, artifacts and recent advances, vol. PM 114. The International Society for Optical Engineering, SPIE Press Monograph; 2003.

[6] Gringauz A, Bar Y. CT image reconstruction. US Patent 68,45,143; 18 January 2005.

[7] Gard MF, SandrikJM. On-line measurement of X-ray tube voltage in a CT system. US Patent 55,30,735; 25 June 1996.

[8] Carrier R, Blais N. Phantom equivalent thicknesses in diagnostic radiology. Phys Med Biol 1987;32(September (9)):1151-7.

[9] Kalender WA. Computed tomography, fundamentals, system technology, image quality, applications. 2nd revised edition Publicis Corporate Publishing; 2005.

[10] Harris CH. Road to RSNA 2008: CT Preview, Auntminnie.com; 29 October 2008. 